\title{
Facebook Rigorous Application Evaluator to Focused on Detecting Malicious Apps on Facebook
}

\author{
Neela Kiranmai \\ Assistant Prof, \\ Dept. of CSE, \\ Sreenidhi Institute of \\ Science and Technology, Hyderabad.
}

\begin{abstract}
Together with 20 billion includes every day, outsider Apps can be a critical reason for the appeal not withstanding addictiveness of Facebook. Unfortunately, digital hoodlums get went to the acknowledgment, the likely of Applying Facebook with respect to scattering malware not withstanding spontaneous mail.More than $13 \%$ of the dataset are normally pernicious. Up to now, the examination by nearby group gives revealing vindictive substance notwithstanding notices. On this report, a large portion of people question the issue: introducing some kind of Facebook programming, can absolutely the majority of people find out on the off chance that it is malignant? This paper focuses in building FRAppE Face book's Thorough Request Evaluator likely the essential device gave to revealing vindictive Facebooks in Facebook. To deliver FRAppE, the vast majority of people use truths acquired just by seeing the submitting conduct of $111 \mathrm{~K}$ Facebook Facebooks watched all through 2.2 zillion clients in Facebook. In the first place, the majority of people distinguish a few qualities that will help every one separate pernicious Facebooks by not dangerous individuals. For instance, the vast majority of people understand that pernicious Facebooks for the most part impart names along to extra Facebooks, thus they generally request a considerable measure less authorizations when contrasted with not destructive Facebooks. Next, influence these sorts of recognizing qualities, a large portion of people exhibit that FR Facebook $\mathrm{E}$ can unquestionably discover pernicious Facebooks alongside $99.5 \%$ dependability, without false pluses and in addition an insignificant false antagonistic rate (4. 1\%). At long last, the vast majority of people look at nature of malicious Facebook Facebooks notwithstanding recognize parts why these Facebooks use keeping in mind the end goal to increase. For some odd reason, the vast majority of people understand that numerous Facebooks intrigue notwithstanding help the other; in the dataset, a large portion of people find 1, 584 Facebooks permitting the infection like dissemination of 3, 723 extra Facebooks as a consequence of their substance. Long haul, the greater part of people perspective FR Facebook $\mathrm{E}$ to be an activity toward building up a private guard dog in regards to Facebooklication examination not with-standing position, trying to caution Facebook clients in front of introducing Facebooks.
\end{abstract}

\section{Keywords}

Content Based Information Retrieval, Online Social Media, Privacy preserving CBIR System.

\section{INTRODUCTION}

In the Internet time, interactive media substance is enormously delivered and disseminated. So as to proficiently find content in a vast scale database, content-based pursuit systems have been created. They are utilized by substance based data recovery [1] frameworks to supplement ordinary watchword based procedures in applications, for example, close copy location, programmed comment, proposal, and so on. In such a run of the mill situation, a client could give a recovery framework an arrangement of criteria or case as a question; the framework returns important data from the database as an answer. As of late, with the rise of new applications, an issue with substance based pursuit has emerged once in a while the inquiry or the database contains protection touchy data. In an organized situation, the parts of the database proprietor, the database client, and the database administration supplier can be taken by various gatherings, who don't as a matter of course trust each other. A security issue emerges when an un trusted party needs to get to the private data of another gathering. All things considered, measures ought to be taken to ensure the comparing data. The primary test is that the inquiry must be performed without uncovering the first question or the database. This propels the requirement for protection safeguarding CBIR frameworks. Security brought early consideration up in biometric frameworks, where the inquiry and the database contain biometric identifiers. Biometric frameworks seldom keep information free, dreading robberies of such exceedingly important information. Correspondingly, a client is hesitant in sending his biometric format free. Traditionally, biometric frameworks [5] depend on cryptographic primitives to ensure the database of formats. In the interactive media area, protection issues as of late developed in substance proposal. With suggestion frameworks, clients are commonly profiled. Profiles are sent to administration suppliers, which send back customized content. Clients are today compelled to believe the administration suppliers for the utilization of their profiles. In spite of the fact that CBIR frameworks have not been broadly sent yet, comparable dangers exist. As of late, the restricted protection model for CBIR was explored [1]. The restricted security setting expect that exclusive the client needs to over the previous decade, online networking (OSM) has stamped its power as one of the biggest data propagators on the Internet. OSN administrations have deled all local, social, and dialect limits, and gave each Internet client on the planet with an equivalent chance to talk, and be listened. Almost $25 \%$ of the total populace utilizes no less than one online networking administration today. 1 People over the globe effectively utilize online networking stages like Twitter and Facebook for spreading data, or finding out about true occasions nowadays. A late study uncovered that online networking movement increments up to 200 times amid significant occasions like decisions, games, or characteristic catastrophes. This swollen movement contains a ton of data about the occasions, but at the same time is inclined to serious misuse like spam, falsehood, and gossip spread, and has along 
these lines drawn extraordinary consideration from the software engineering research group. Since this surge of data is created and devoured continuously, and by basic clients, it is difficult to remove valuable and noteworthy substance, and later out undesirable food. Twitter, specifically, has been generally concentrated on by analysts amid genuine occasions. Be that as it may, few studies have taken a gander at the substance spread on online networking stages other than Twitter to concentrate genuine occasions [Chen and Roy 2009; Hille and Bakker 2013; Osborne et al. 2012]. Shockingly, there has been little work on contemplating content on Facebook amid true occasions, which is five times greater than Twitter as far as the quantity of month to month dynamic clients. Scope of examination endeavors which would investigate vindictive substance spread on Facebook amid occasions. Specifically, we take a gander at three particular territories, viz. a) the Facebook social diagram, b) assault and identification procedures as for malevolent substance on Facebook, and c) investigation of occasions utilizing online networking information. At that point, we take a gander at the different constraints that Facebook postures, which makes occasion investigation, and discovery of vindictive substance on this system a difficult issue. Towards the end, we talk about the suggestions and exploration crevices in recognizing and dissecting pernicious client created content on Facebook amid occasions.

\section{PROBLEM STATEMENT}

At present, pernicious applications regularly do exclude a classification, organization, or portrayal in their application rundown. To identify the malignant face book applications which may influences to client's private data on his/her profile. As we see client did not get much data about application expect name of that application while introducing accordingly no security accessible on Facebook.

\section{MALICIOUS CONTENT ON FACEBOOK}

The prevalence and compass of Facebook has additionally pulled in a considerable measure of spam, phishing, malware, and different sorts of malevolent movement. Assailants draw casualties into tapping on malignant connections indicating outside sources, and in proficient their system. These connections can be spread either through individual messages, or through divider posts. To accomplish greatest perceivability, aggressors like to post interfaces openly. Commonly, an aggressor starts the assault by posting images with consideration getting sneak peeks, which brief clients to like, share, or remark on them so as to view them. The activities of preferring, remarking or sharing spread these pics into the casualty's system. Once the pic is spread, the casualty is diverted to a malignant site, which can promote taint her PC, or companions system through phishing, malware, or spyware. This phishing page requests that the casualty impart this video to their companions so as to view it. In any case, once the casualty shares this video, the page sidetracks to an irregular ad page. The video relating to the sneak peak/thumbnail appeared in the post does not really exist. Numerous different sources have referred to such case of tricks and pernicious posts on Facebook in the previous couple of years. 11, 12 notwithstanding phishing tricks, different noxious movement on Facebook incorporates spontaneous mass notice, photograph labeling, post labeling, private/talk messages and so on. Naturally, a client will probably react to a message or post from a Facebook companion than from a more interesting, along these lines making this social spam a more compelling circulation component than customary email. This expanded defenselessness to such sort of spam has provoked specialists to study, and battle social spam and different noxious action on Facebook. We now take a gander at the different assault and location strategies that have been utilized as a part of the past to distinguish and spread noxious substance on Facebook individually.

\subsection{Attack procedures}

Keeping in mind the end goal to recognize and contain noxious posts on Facebook, or any OSM, it is key to investigate and comprehend the systems that are, or can conceivably be conveyed by assailants to spread such substance. depicted how Facebook can be abused and changed over into an assault stage, with a specific end goal to increase some touchy information, which can finish a flawless assaulting genius le against a client. Writers made a Facebook application for show purposes that at first glance was a basic application, however on the foundation it gathered valuable information. This application executed pernicious code on the casualty's program, and gathered the IP location of the client casualty, the program form, the OS stage and whether some particular ports are open or shut. This information was then transmitted to the creators over email. Writers likewise called attention to that their application was filed on the principle rundown of Facebook applications, regardless of the way that the depiction of application unmistakably expressed that it was producing pernicious exchange, and had been made for entrance testing purposes. Huber et al. displayed a companion in the center assault through capturing session treats. Creators clarified how it was conceivable to imitate the casualty utilizing this procedure, and cooperate with the system without legitimate approval. Be that as it may, this system was proposed in 2011, when utilizing HTTPS to interface with the site was discretionary. 13 Post 2013, all correspondence on Facebook utilizes encryption (HTTPS) of course, which implies that such assaults are not any more conceivable. Fan et al. [Fan and Yeung 2010] proposed an infection model in light of the application system of Facebook. Writers likewise displayed the infection engendering with an email infection demonstrate and looked at the practices of infection spreading in Facebook and email system. Their discoveries uncovered that while Facebook gives a stage to application engineers, it additionally gives the same opportunity to infection spreading. Actually, the infection was found to spread speedier on the Facebook system if clients invest more energy in it. The aftereffect of their recreation demonstrated that, despite the fact that a malignant Facebook application draws in just a couple of clients in the first place, it can at present spread quickly. That is on account of clients may believe their companions of Facebook and introduce the vindictive application. It is essential to comprehend that notwithstanding the methods portrayed over, a vast extent of assaults on Face book, and even other person to person communication stages, make utilization of social building. This is clear since it is difficult to start the spread of a pernicious bit of substance on a system with no human contribution. Assailants bait casualties into utilizing malignant applications, clicking vindictive connections and sharing bits of substance, and at times, even put on a show to give different sorts of advantages consequently. Since these assaults are all around created as a rule, it turns out to be hard for a honest to goodness client to have the capacity to understand the consequences of her activities. We now take a gander at the different procedures that have been proposed to identify pernicious substance on the Facebook informal organization. 


\subsection{Detection strategies}

Facebook has its own invulnerable framework to defend its clients from undesirable, malevolent substance. Analysts at Facebook assembled and sent a sound, versatile, and extensible ongoing framework to ensure their clients and the social chart. This framework performs continuous checks and orders on each read and compose.

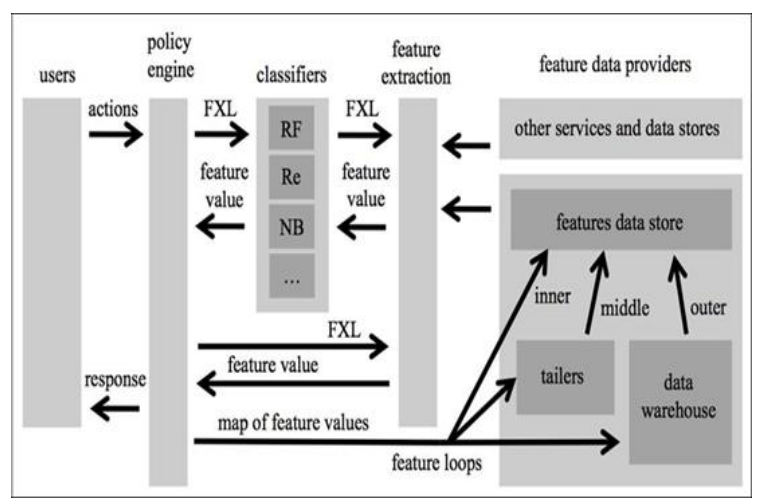

Keeping in mind the end goal to distinguish and contain noxious posts on Facebook, or any OSM, it is crucial to investigate and comprehend the methods that are, or can possibly be sent by aggressors to spread such substance. To this end, Patsakis et al. portrayed how Facebook can be abused and changed over into an assault stage, keeping in mind the end goal to increase some delicate information, which can finish a flawless assaulting ace le against a client. Writers made a Facebook application for show purposes that at first glance was a basic application, however on the foundation it gathered valuable information. This application executed malignant code on the casualty's program, and gathered the IP location of the client casualty, the program form, the OS stage and whether some particular ports are open or shut. This information was then transmitted to the creators over email. Writers likewise called attention to that their application was recorded on the primary rundown of Facebook applications, in spite of the way that the depiction of application obviously expressed that it was producing pernicious exchange, and had been made for entrance testing purposes. Huber et al. displayed a companion in-the-center assault through seizing session treats. Creators clarified how it was conceivable to imitate the casualty utilizing this procedure, and collaborate with the system without legitimate approval. Be that as it may, this strategy was proposed in 2011, when utilizing HTTPS to interface with the site was discretionary. 13 Post 2013, all correspondence on Facebook utilizes encryption (HTTPS) naturally, which implies that such assaults are not any more conceivable. Fan et al. [Fan and Yeung 2010] proposed an infection model in view of the application system of Facebook. Writers additionally demonstrated the infection engendering with an email infection show and looked at the practices of infection spreading in Facebook and email system. Their discoveries uncovered that while Facebook gives a stage to application designers, it additionally gives the same opportunity to infection spreading. Truth be told, the infection was found to spread quicker on the Facebook system if clients invest more energy in it. The consequence of their reproduction demonstrated that, despite the fact that a vindictive Facebook application draws in just a couple of clients first and foremost, it can in any case spread quickly. That is on the grounds that clients may believe their companions of Facebook and introduce the malignant application. It is critical to comprehend that notwithstanding the methods portrayed over, an expansive extent of assaults on Facebook, and even other long range informal communication stages, make utilization of social building. This is obvious since it is difficult to start the spread of a noxious bit of substance on a system with no human association. Aggressors bait casualties into utilizing malevolent applications, clicking pernicious connections, and sharing bits of substance, and at times, even put on a show to give different sorts of advantages consequently. Since these assaults are all around made as a rule, it turns out to be hard for a true blue client to have the capacity to understand the aftereffects of her activities. We now take a gander at the different procedures that have been proposed to distinguish malignant substance on the Facebook interpersonal organization.

Facebook itself has affirmed spam as a significant issue, and found a way to decrease spam content in clients, newsfeed as of late [Owens and Turitzin 2014]. Distinguishing spam on Facebook, in any case, clearly remains a difficult issue. Regardless of Facebook having an elite invulnerable arrangement of their own [Stein et al. 2011], clients still experience a tremendous number of spam and vindictive substance on general premise. Existing ways to deal with distinguish spam in other online social networking administrations like Twitter [Benevenuto et al. 2010; Grier et al. 2010; McCord and Chuah 2011; Wang 2010], can't be specifically ported to Facebook because of different issues. These incorporate the general population inaccessibility of basic bits of data like ace le, and system data, age of the record, no restriction on post length, and so forth. There exists critical need to study spam content on Facebook, and create procedures to recognize it fittingly, and consequently.

\section{THE PROPOSED FRAME WORK}

In this work, FRAppE app is to be created, a suite of effective characterization procedures for distinguishing whether an application is pernicious or not. To manufacture FRAppE, we have to utilize information from MyPageKeeper. To construct FRAppE, we utilize information from MyPage Keeper, a security application in Facebook that screens the Facebook profiles of 2.2 million clients. We dissect $111 \mathrm{~K}$ applications that made 91 million posts more than nine months. This is seemingly the principal thorough study concentrating on malevolent Facebook applications that spotlights on evaluating, profiling, and comprehension pernicious applications, and blends this data into a powerful identification approach. Two components i.e. classifiers to distinguish the vindictive applications FRAppELite and FRAppE . In first classifier it recognizes the underlying level recognition e.g. applications personality number, name and source and so on and in second level location the real recognition of noxious application has been finished.

\section{CONCLUSION}

In this study, investigations have been done on different exploration endeavors towards investigating the Facebook system, examining pernicious substance on it, and breaking down occasions on online networking as a rule. The point of this study was to take a gander at important writing, which could help in concentrating on and fighting pernicious client produced content spread on Facebook amid occasions. So as to keep this review centered, An assortment of potentially pertinent examination ranges including identification of traded off/fake records, and sybil hubs in the Facebook system, discovery of spam on other interpersonal organizations like Twitter, validity/reliability of data of client created substance, and occasion location in online networking ,have not been covered. Additionally took a gander at the different 
difficulties and restrictions postured by Facebook. Aside from specialized confinements, there exist different exploration holes in existing writing, which are yet to be tended to and investigated.

\section{REFERENCES}

[1] Besmer, H. R. Lipford, M. Shehab, and G. Cheek. Social applications: exploring a more secure framework. In SOUPS, 2009.

[2] C.-C. Chang and C.-J. Lin. LIBSVM: A library for support vector machines. ACM Transactions on Intelligent Systems and Technology, 2, 2011.

[3] P. Chia, Y. Yamamoto, and N. Asokan. Is this app safe? A large scale study on application permissions and risk signals. In WWW, 2012.

[4] F. J. Damerau. A technique for computer detection and correction of spelling errors. Commun. ACM, 7(3), Mar. 1964.

[5] H. Gao, Y. Chen, K. Lee, D. Palsetia, and A. Choudhary. Towards online spam filtering in social networks. In NDSS, 2012.

[6] H. Gao, Y. Chen, K. Lee, D. Palsetia, and A. Choudhary. Towards online spam filtering in social networks. In NDSS, 20124. J. King, A. Lampinen, and A. Smolen. Privacy: Is there an app for that? In SOUPS, 2011

[7] J. King, A. Lampinen, and A. Smolen. Privacy: Is there an app for that? In SOUPS, 2011.

[8] Lee, J. Caverlee, and S. Webb. Un-covering social spammers: social honey pots machine learning. In SIGIR, 2010
[9] Lee and J. Kim. Warningbird: Detecting suspicious urls in twitter stream. In NDSS, 2012.

[10] Y. Liu, K. P. Gummadi, B. Krishna murthy, and A. Mislove. Analyzing face book privacy settings: user expectations vs. reality. In IMC, 2011.

[11] Makridakis, E. Athanasopoulos, S. Antonatos, D. Antoniades, S. Ioannidis, and E. P. Markatos. Understanding the behavior of malicious applications in social networks. Netwrk. Mag. of Global Internet wkg., 2010.

[12] M. S. Rahman, T.-K. Huang, H. V. Madhyastha, and M Faloutsos. Efficient and Scalable Socware Detection in Online Social Networks. In USENIX Security, 2012.

[13] T. Stein, E. Chen, and K. Mangla. Facebook immune system. In Proceedings of the 4th Workshop on Social Network Systems, 2011.

[14] G. Stringhini, C. Kruegel, and G. Vigna. Detecting spammers on social networks. In ACSAC, 2010

[15] K. Thomas, C. Grier, J. Ma, V. Paxson, and D. Song. Design and Evaluation of a Real-Time URL Spam Filtering Service. In Proceedings of the IEEE Symposium on Security and Privacy, 2011.

[16] N. Wang, H. Xu, and J. Grossklags. Third-party apps on face book: privacy and the illusion of control. In CHIMIT, 2011

[17] Yang, R. Harkreader, and G. Gu. Die free or live hard? empirical evaluation and new design for fighting evolving n twitter spammers. In RAID, 2011. 